\title{
Matrix-based analysis of digital images:
}

\author{
application to goniophotometric
}

measurements with variable referential

\author{
M. Andersen ${ }^{a, *}$, \\ ${ }^{a}$ Swiss Federal Institute of Technology (EPFL), Solar Energy and Building Physics \\ Laboratory (LESO-PB), Building LE, 1015 Lausanne, Switzerland
}

\begin{abstract}
Digital video based luminance mapping systems require the establishment of a precise relation between the considered spatial referential and the associated pixel coordinates on the image. Such a relation may vary with the measurement conditions, and an adjustment of the geometric calibration therefore becomes necessary. In this paper, a methodology of adapting the image calibration according to the referential transformations is proposed, based on the use of a set of matrices individually associated to each spatial coordinate. These matrices are modified according to analytical conversions in order to define the actual measurement situation. The description of this approach is given through an application example on a recent bidirectional goniophotometric device, based on digital imagery, proving the consistency of the adopted methodology.
\end{abstract}

Key words: Digital imaging (CCD), Image processing, Matrix array, Photometry, Geometric calibration, Bidirectional goniophotometer, BTDF, BRDF 


\section{Introduction}

Many photometric measurement facilities have chosen to take benefit from the use of digital imaging techniques $(1 ; 2 ; 3 ; 4 ; 5 ; 6 ; 7 ; 8 ; 9 ; 10 ; 11 ; 12)$, and the latter have proven to be efficient and reliable, allowing considerable improvements in speed and flexibility. To extract photometric quantities from numeric images provided by a Charge-Coupled Device (CCD) camera, several calibration phases have to be achieved, amongst which we find: the spectral sensitivity correction, that has to fit the photopic curve $\mathrm{V}(\lambda)$ (human eye spectral sensitivity), the conversion from pixel values into associated luminances, which depends on the considered integration time (photometric calibration), and the link between pixel coordinates and associated positions in real space (geometric calibration), in order to know to what exact direction the luminance given by the calibrated pixel value corresponds.

In some cases, it is possible to keep a unique referential, as for sky-scanners for instance (8): the sky luminance distribution can be associated to a fixed spatial referential, the sky vault being considered as an illuminating hemispherical surface. The only spatial parameters that may vary could be obstructions to the visualization of the entire sky dome, due to buildings or mountains for instance, or to mechanical components in the field of vision.

However, in many cases, the referential can change, especially when the transformations depend on the observer's position, on the surrounding dimensions, on the emission point, etc. In this case, it becomes difficult to adapt the

\footnotetext{
* Corresponding author. Tel: +41-21-693-4551; fax: +41-21-693-2722.

Email address: marilyne.andersen@epfl.ch (M. Andersen).

$U R L:$ http://lesowww.epfl.ch/doctorants/andersen/(M. Andersen).
} 
geometric calibration analytically, because it may depend on non-linear parameters, like lens optical aberrations for example. A solution could be the establishment of several geometric calibrations, each one being adapted to certain measurement conditions. But this method still remains fixed, and may become either insufficient, as there always will exist new conditions, or too approximate, as all possible measurement situations must be gathered into a finite number of discrete conditions intervals.

A way to solve this problem appears when a well-known spatial referential undergoes transformations that can be described analytically, without the need of deducing spatial parameters from the recognition of patterns on captured images $(13 ; 14)$. The method consists of using a set of matrices, whose dimensions are equal to the pixel resolution of the digital images, and which are composed of spatial coordinates describing the system, each coordinate being associated to a different matrix. The referential transformations can thus be applied by calculating the corresponding modifications on the matrices, which are then taken as a basis to adapt the image geometric calibration.

In order to extensively illustrate this approach, we will use a concrete case of using digital imaging for photometric measurements, where referential transformations are present: the bidirectional goniophotometer developed at the Solar Energy and Building Physics Laboratory (LESO-PB, EPFL) for transmission (BTDF) and reflection (BRDF) measurements, that allows to assess the so-called Bidirectional Transmission (Reflection) Distribution Function, abbreviated BT(R)DF. This function is defined as the ratio between the emerging luminance $L_{2}$ of a surface element in a given direction and the illuminance $E_{1}$ on the sample plane, and is expressed in $\mathrm{Cd} \cdot \mathrm{m}^{-2} \cdot \mathrm{lux}^{-1} \mathrm{or} \mathrm{sr}^{-1}$. Details on its 
calibration procedures, image and data processing and results can be found in $(1 ; 15)$.

\section{$2 \mathrm{BT}(\mathrm{R}) \mathrm{DF}$ assessment based on digital imaging}

The functioning principle of the bidirectional goniophotometer developed at the LESO-PB / EPFL and based on digital imaging techniques is the following: the light emerging from the sample is reflected by a diffusing triangular panel towards a CCD camera, which provides a picture of the screen in its entirety; the camera is used as a multiple-points luminance-meter and calibrated accordingly. Within six positions of the screen and camera around the sample (each separated by a $60^{\circ}$ rotation from the next one), a complete investigation of the transmitted or reflected light is achieved. This method, illustrated in transmission on Figure 1(a), allows to overcome the two main draw-backs of the conventional - and maybe more intuitive - approach that consists of scanning the emerging light flux distribution by moving a sensor from one position to the other. Indeed, it reduces the time needed to perform a bidirectional measurement to a few minutes per incident direction instead of several hours, which is a critical parameter in $\mathrm{BT}(\mathrm{R}) \mathrm{DF}$ assessment as about a hundred incident directions are usually required; at the same time, it allows a continuous investigation of the transmitted (reflected) light distribution, only limited in resolution by the pixellisation of the digital images, while the scanning process provides a discrete information, where the risk of missing a narrow feature is never nil.

The spatial referential is given in spherical coordinates. The light source remaining fixed, the incident direction is determined by tilting the device (and 
hence the sample plane) around a horizontal axis and rotating the sample around its normal. As shown on Figure 1(b) for BTDFs, the referential origin is placed on the sample itself and the directions are defined by their polar angles: altitude $\theta_{i}$ is comprised between $0^{\circ}$ and $90^{\circ}$ and azimuth $\phi_{i}$ is comprised between $0^{\circ}$ and $360^{\circ}$, where index i indicates whether the angle is related to the incident $(\mathrm{i}=1)$ or emerging $(\mathrm{i}=2)$ direction (either transmitted or reflected). The base plane for incidence (i.e. where $\theta_{1}=90^{\circ}, \phi_{1} \in\left[0^{\circ} ; 360^{\circ}[\right.$ ) is given by the external sample interface, which is fixed; as far as the base plane for emerging light is concerned $\left(\theta_{2}=90^{\circ}, \phi_{2} \in\left[0^{\circ} ; 360^{\circ}[)\right.\right.$, it coincides with the former for reflected light, but is given by the internal sample interface for transmitted light, that will thus be shifted according to the sample thickness, as explained in Section 4. Besides, the origin axes $\phi_{i}=0^{\circ}, i=1,2$, whose vectors are collinear for incidence and emergence, are bound to the sample itself; a rotation of the latter will therefore induce a referential transformation, discussed in Section 4 as well.

Fig. 1. Assessment method and polar coordinates of the Bidirectional Transmission Distribution Function (defined likewise in reflection).

At each screen position, digital images are captured at several integration intervals and greyscale levels are transformed into corresponding luminances and divided by the simultaneously measured illuminance $E_{1}\left(\theta_{1}\right)$; the superposition of different integration intervals for the same luminous situation improves the accuracy of luminance measurements (32 bits values) and avoids over-exposure (saturation) and/or under-exposure in presence of high luminance dynamics. Compensating distance and light tilting effects, the obtained "screen" luminances $L_{\text {screen }}\left(\theta_{1}, \phi_{1}, \theta_{2}, \phi_{2}\right)$ are finally converted into Bidirectional Transmission (Reflection) Distribution Functions (BT(R)DFs) through Equation (1); 
gathering the results of the six positions of the screen and camera system, the emerging light distribution is fully known $(1 ; 15)$.

$$
B T(R) D F\left(\theta_{1}, \phi_{1}, \theta_{2}, \phi_{2}\right)=\frac{\pi}{\rho_{\text {screen }}} \cdot \frac{d^{2}\left(\theta_{2}, \psi_{2}\right)}{A \cdot \cos \alpha \cdot \cos \theta_{2}} \cdot \frac{L_{\text {screen }}\left(\theta_{1}, \phi_{1}, \theta_{2}, \phi_{2}\right)}{E_{1}\left(\theta_{1}\right)}(1)
$$

where $\rho_{\text {screen }}$ is the screen reflection coefficient, $A$ the illuminated area, $d$ the distance between the sample and the screen along direction $\left(\theta_{2}, \phi_{2}\right)$, and $\alpha$ is defined as the angle between the direction $\left(\theta_{2}, \phi_{2}\right)$ and the normal to the screen (Equation (2)) (15); it is represented on Figure 3 below.

$$
\alpha\left(\theta_{2}, \psi_{2}\right)=\arccos \left(\cos \Theta_{0} \cdot\left(\frac{2}{\sqrt{3}} \cdot \sin \theta_{2} \cdot \cos \psi_{2}+\cos \theta_{2}\right)\right)
$$

where $\Theta_{0}$ is the angle between the projection panel and the main platform $\left(\Theta_{0}=\arctan \frac{2}{\sqrt{3}} \cong 49.1^{\circ}\right.$ : see Figure 3$)$, chosen so that an orthogonal projection of the screen on the main platform leads to an equilateral triangle.

In order to extract usable data from the calibrated images, the emergence hemisphere is split into angular sectors around the considered directions $\left(\theta_{2}, \phi_{2}\right)$, thus determining an averaging grid for BTD $(\mathrm{R})$ Fs with regular intervals $\left(\Delta \theta_{2}, \Delta \phi_{2}\right)$ (16). The emerging luminances being here measured on a projection screen, the latter must be divided into a certain grid of averaging sectors that depends on the most suitable angular intervals. Two examples are shown on Figures $2(\mathrm{a})$ and $2(\mathrm{~b})$, for intervals $\left(\Delta \theta_{2}, \Delta \phi_{2}\right)$ equal to $\left(5^{\circ}, 5^{\circ}\right)$ and $\left(10^{\circ}, 15^{\circ}\right)$ respectively.

Fig. 2. Division of the screen measurement area into discretisation sectors, differentiated by grey level gaps. 
To remain consistent with the possible divergence of rays reaching a given point, the angular resolution has to be chosen according to the sample size (16), involving a need for flexibility in the angular intervals of the averaging grid, moreover enhanced by the multiplicity of BT(R)DF applications (industry, architecture, daylighting simulation tools) with very different requests in accuracy and emerging light distribution details. These requirements for flexibility strengthen the need for fine adaptations of the spatial referential, presented in Section 4.

\section{Reference frame configuration}

The determination of the relationship between the spherical co-ordinates $\left(\theta_{2}, \phi_{2}\right)$ defined around the sample (see Figure ??) and the pixel co-ordinates $(X, Y)$ on the captured image must be considered in three steps:

- first ensure a fixed position of the screen area on the digital image by adjusting a triangular Area Of Interest (AOI) to the pixels corresponding to the actual screen detection area (15);

- then relate the screen's pixels to their corresponding emerging polar angles $\left(\theta_{2_{\text {Ref }}}, \psi_{2}\right)$ for a reference configuration and an arbitrary screen position;

- finally, determine the hemispherical polar angles $\left(\theta_{2}, \phi_{2}\right)$ accounting for the current screen position, the sample thickness $e_{s}$ and the incident azimuth $\phi_{1}$. 


\subsection{Spatial referential definition}

As illustrated by Figure 3, the base plane chosen to define the polar coordinates referential was the plane parallel to the sample and comprising the detection area base. $x$ and $y$ are measured according to Figure 3 along the detection area base and its central axis. Altitude $\theta_{2_{\text {Ref }}}$ is the angle between the considered direction and the normal to the sample plane; $\psi_{2}$ is its azimuth angle, projected on the same plane and comprised between $-30^{\circ}$ and $+30^{\circ}$ (zero along the central axis of the screen). As the detection area base is shifted by $g=7.5 \mathrm{~cm}$ from the external (incident) sample plane for mechanical reasons (rotating ring structure and movements), this reference configuration actually corresponds to a sample thickness $e_{s}=7.5 \mathrm{~cm}$ for transmission measurements; such a thickness indeed shifts the internal (emerging) sample interface plane down to the detection area's base level.

Fig. 3. Relationship between polar co-ordinates $\left(\theta_{2}, \psi_{2}\right)$ and reference coordinates $\left(\theta_{2_{\text {Ref }}}, \psi_{2}\right)$, combined to the associated cartesian screen co-ordinates $(x, y)$.

The cartesian coordinates $(x, y)$ on the screen detection area can be expressed as functions of their associated polar angles $\left(\theta_{2_{\text {Ref }}}, \psi_{2}\right)$ through Equations (3), deduced from trigonometric considerations:

$$
\begin{aligned}
& x=\frac{H}{\sin \Theta_{0}} \cdot \frac{1}{\frac{2}{\sqrt{3}} \cdot \tan \theta_{2_{\mathrm{Ref}}} \cdot \cos \psi_{2}+1} \\
& y=H \frac{\sin \psi_{2}}{\frac{2}{\sqrt{3}} \cdot \cos \psi_{2}+\cot \theta_{2_{\mathrm{Ref}}}} \\
& d_{\text {Ref }}=\frac{H}{\frac{2}{\sqrt{3}} \cdot \sin \theta_{2_{\mathrm{Ref}}} \cdot \cos \psi_{2}+\cos \theta_{2_{\mathrm{Ref}}}}
\end{aligned}
$$

where $H=115 \mathrm{~cm}$ is the "vertical" distance from the detection area apex to 
the base plane and $d_{\text {Ref }}$ is the distance between the referential origin and the screen plane along direction $\left(\theta_{2_{\text {Ref }}}, \psi_{2}\right)$.

In order to directly incorporate image aberrations, avoiding the necessity of correcting image distortions independently, a set of points of known polar coordinates $\left(\theta_{2_{\text {Ref }}}, \psi_{2}\right)$ was drawn on the projecting screen, for each one to be manually located at a particular pixel and therefore associated to its coordinates $(X, Y)(1)$.

\subsection{Matrix representation}

Based on the obtained arrays and on Equations (3), three $685 \times 488$ matrices $M_{\theta_{2} \text { Ref }}, M_{\psi_{2}}$ and $M_{d_{\text {Ref }}}$ were created, of dimensions equal to the image size and represented on a pixel greyscale on Figures 4(a), 4(b) and Figure 4(c). Their coefficients are respectively equal to angles $\theta_{2_{\text {Ref }}}$ and $\psi_{2}$ and distance $d_{\text {Ref }}$ associated to the corresponding image pixels. Out of the screen detection area, the matrix coefficients are set to $N a N$ (Not A Number).

These three matrices are used as a calculation basis for referential transformations (see Section 4); for a better visual display on Figure 4, the 0 to 255 greyscale was fitted to each parameter's angular or metric range individually. Their respective values vary from $0^{\circ}$ to $90^{\circ}$ for $\theta_{2_{\text {Ref }}},-30^{\circ}$ to $30^{\circ}$ for $\psi_{2}$, and $753 \mathrm{~cm}$ to $115 \mathrm{~cm}$ for $d_{\text {Ref. }}$ Pixels out of the screen area ( $N a N$ in the matrices) were arbitrarily set to 0 .

Fig. 4. Digital representation of reference matrices (for a quantitative analysis, they can be compared against the contour plots of Figures 2(a) and 2(b). 


\section{Reference frame transformations}

As the referential is linked to the sample itself, the emerging polar angles $\left(\theta_{2}, \phi_{2}\right)$ have to be adapted to two sample parameters:

- in transmission mode, to the sample thickness $e_{s}$, as the emerging interface determines the referential base plane (in reflection mode, the single configuration corresponds to a zero thickness, as incident and emerging interfaces are coplanar); a given point on the screen (or a same pixel on the image) thus corresponds to varying $\theta_{2}$ angles, depending on $e_{s}$;

- in both modes, to the incident azimuth $\phi_{1}$; indeed, as the sample's tilt and orientation determine the incident direction, the azimuth origin axis is rotated around the normal to the sample according to angle $\phi_{1}$; hence, a given point on the screen (a same pixel) can also correspond to different $\phi_{2}$ values.

\subsection{Translation with sample thickness}

As illustrated by Figure 3, the correspondence between $\left(\theta_{2_{\text {Ref }}}, \psi_{2}\right)$ and $\left(\theta_{2}, \psi_{2}\right)$ depends on different fixed quantities (gap $g=7.5 \mathrm{~cm}$, distance $H$, angle $\Theta_{0}$ ) and on the variable sample thickness $e_{s}$, that can induce significant differences in the altitude $\theta_{2}$ to which a given pixel corresponds (e.g. up to $2^{\circ}$ between a null thickness and a configuration where $e_{s}=3 \mathrm{~cm}$ ).

In fact, only $\theta_{2}$ varies with $e_{s}$, whereas $\psi_{2}$ remains unchanged. Defining it as a function of $\theta_{2_{\text {Ref }}}, \psi_{2}$ and $d_{\text {Ref }}$ using Equations (??) and (3), Equation (4) can 
be written:

$$
\theta_{2}=\arcsin \frac{d_{\mathrm{Ref}} \cdot \sin \theta_{2_{\mathrm{Ref}}}}{\sqrt{\left(d_{\mathrm{Ref}} \cdot \cos \theta_{2_{\mathrm{Ref}}}+\left(g-e_{s}\right)\right)^{2}+\left(d_{\mathrm{Ref}} \cdot \sin \theta_{2_{\mathrm{Ref}}}\right)^{2}}}
$$

where the distance $d$ between the sample center (in the emerging interface) and the screen is equal to the denominator in Equation (4).

Matrices $M_{\theta_{2}}$ and $M_{d}$ were created according to the sample thickness $e_{s}$ using matrices $M_{\theta_{2_{\text {Ref }}}}, M_{\psi_{2}}$ and $M_{d_{\text {Ref }}}$ and Equation (4) (the arithmetic operations being applied to each of their coefficients individually). Together with $M_{\psi_{2}}$, they provide the essential parameters for the creation of the averaging grid (see Section 5) as well as for compensating distance and light tilt effects on acquired images (creation of a correction factor matrix, see Equation (6)), to achieve pixel-level accurate BT(R)DF data.

\subsection{Rotation with azimuth angle of incident beam}

As illustrated in Figure 5, changing the incident azimuth $\phi_{1}$ is materialized by a rotation of the sample, to which the output referential is linked. It is therefore essential to know the exact position of the azimuth origin axis, and first of all the screen position number $p_{0}$ on which it is projected, fully determined by the $\phi_{1}$ parameter.

$p_{0}$ is defined as the screen position comprising the origin axis for $\phi_{2}$. The exact position of this axis is given by $\psi_{2_{0}}\left(\phi_{1}, \Delta \phi_{2}\right)$, equal to the $\psi_{2}$ value on screen position $p_{0}$ at which it is projected. $\psi_{2_{0}}\left(\phi_{1}, \Delta \phi_{2}\right)$ is comprised between $-30^{\circ}$ and $+30^{\circ}$ and equals zero only if $\phi_{1}=\mathrm{N} \cdot 60^{\circ}$. 
The relationship between $\phi_{2}$ and $\psi_{2}$ can therefore be expressed as follows:

$$
\phi_{2}=\left(\Delta p_{0} \cdot 60^{\circ}+\psi_{2}-\psi_{2_{0}}+360^{\circ}\right) \bmod 360^{\circ}
$$

where $\Delta p_{0}$ is the number of screen positions separating $p_{0}$ from the current position $p$, according to the screen numbering order of Figure 5.

Fig. 5. Orthogonal projection view of the sample orientation superimposed on the six screen positions.

\section{Matrix-based construction of $\mathrm{BT}(\mathrm{R}) \mathrm{DF}$ averaging grid}

As mentioned above, matrices $M_{\theta_{2}}$ and $M_{\psi_{2}}$ serve as a basis for creating $\mathrm{BT}(\mathrm{R}) \mathrm{DF}$ averaging grids according to the chosen angular intervals $\left(\Delta \theta_{2}, \Delta \phi_{2}\right)$.

$\Delta \theta_{2}$ can be chosen in-between $0.5^{\circ}$ and $90^{\circ}$ (with a minimal step of $0.1^{\circ}$ ); $\Delta \phi_{2}$ must be a divisor of $60^{\circ}$. Such constraints avoid problems inherent to resolutions going down to the image pixellisation itself and allow an analysis of the six screen images according to the same individual grid.

\subsection{Grid transformations}

As $\theta_{2}$ values only depend on the sample thickness $e_{s}$, the different fringes delimited by $N \cdot \Delta \theta_{2}-\frac{1}{2} \Delta \theta_{2}$ and $N \cdot \Delta \theta_{2}+\frac{1}{2} \Delta \theta_{2}\left(N=0 \ldots \frac{90}{\Delta \theta_{2}}\right)$ can be easily calculated from $M_{\theta_{2}}$ and $\Delta \theta_{2}$.

Figure 6 shows the superposition of an averaging grid corresponding to $e_{s}=g=7.5 \mathrm{~cm}$ 
(reference configuration, grid sectors delimited by drawn boundaries) over the pattern of sectors associated to $e_{s}=0$ (greyscale level pattern). In both cases, the averaging intervals $\left(\Delta \theta_{2}, \Delta \phi_{2}\right)$ are equal to $\left(10^{\circ}, 15^{\circ}\right)$. This figure also outlines the effect of increasing sample thickness on the $(X, Y) \leftrightarrow\left(\theta_{2}, \psi_{2}\right)$ relationship, stronger for growing values of $\theta_{2}$.

Fig. 6. Superimposed averaging grids with $\left(\Delta \theta_{2}, \Delta \phi_{2}\right)=\left(10^{\circ}, 15^{\circ}\right)$ for $e_{s}=7.5 \mathrm{~cm}$ (sectors borders) and $e_{s}=0$ (greyscale level pattern).

As far as $\phi_{2}$ and $\Delta \phi_{2}$ values are concerned, the averaging grid has to be understood as a splitting of the whole emerging hemisphere, probably presenting several sectors that overlap two screen positions. All six being invariants, the $\phi_{1}$ rotation of the sample (Figure 5) induces a simultaneous revolution of the averaging grid, illustrated by Figure 7(a). Generally speaking, any sector is delimited in azimuth by intervals $\left[N \Delta \phi_{2}-\frac{1}{2} \Delta \phi_{2} ; N \Delta \phi_{2}+\frac{1}{2} \Delta \phi_{2}\left[\left(N=0 \ldots \frac{360}{\Delta \phi_{2}}\right)\right.\right.$, wherever $\phi_{2}$ finds its origin axis.

From the averaging grid's configuration point of view, two types of situations must be distinguished: either $\phi_{1}$ is a multiple of $\Delta \phi_{2}$, or not.

In the first case, the averaging sectors arrangement remains unchanged from the default grid applied when $\phi_{1}=0^{\circ}$, although the azimuth angles $\phi_{2}$ to which they correspond will depend on the exact value of $\phi_{1}$ (see Figure 7(a)).

In the case $\phi_{1}$ is not a multiple of $\Delta \phi_{2}$, the shift in azimuth does not correspond to entire grid steps anymore, which induces the general grid to be completely different from the default one: sectors $\phi_{2}$ and $\psi_{2}$ do not match, as shown on 
Figure 7(b). A new individual grid must therefore be built to subdivise the screen into appropriate averaging sectors. Thanks to the mentioned conditions imposed on $\Delta \phi_{2}$, it can be kept unique for the six screen positions, with analytical adaptations to be established for the corresponding sectors coordinates.

Fig. 7. Schematised global averaging grid for $\left(\Delta \theta_{2}, \Delta \phi_{2}\right)=\left(15^{\circ}, 15^{\circ}\right)$ and sectors' polar coordinates.

Besides, it can be noted that the effective shift between any averaging grid and the corresponding default one is actually equal to $\phi_{1} \bmod \Delta \phi_{2}$; two configurations presenting different $\phi_{1}$ but a same value for $\phi_{1} \bmod \Delta \phi_{2}$ in consequence only need one new grid, the conversion from $\psi_{2}$ to $\phi_{2}$ being obtained through Equation (5).

As illustrated on Figure 8, the grouping of pixels on the screen image undergoes a rotation according to the general grid shift. In practice, a $\phi_{1} \bmod \Delta \phi_{2}$ term (equal to $5^{\circ}$ for the case displayed on the figure) is added to the $M_{\psi_{2}}$ matrix and the pixels grouping takes place based on the resulting "shifted" matrix.

Fig. 8. Superimposed averaging grids with $\left(\Delta \theta_{2}, \Delta \phi_{2}\right)=\left(10^{\circ}, 15^{\circ}\right)$ for $\phi_{1}=50^{\circ}$ (sectors borders) and $\phi_{1}=0^{\circ}$ (greyscale level pattern), displaying a $5^{\circ}$ shift in azimuth. 


\subsection{Binary matrices operations for pixel grouping}

Before creating individual $\left(\Delta \theta_{2}, \Delta \phi_{2}\right)$ clusters of matrix coefficients, the latter are gathered in $\Delta \theta_{2}$ and $\Delta \phi_{2}$ groups: using matrices $M_{\theta_{2}}$ and $M_{\psi_{2}}$, two sets of binary matrices are created:

- $M_{\{\theta\}}$ for the grouping of altitude coefficients within $\Delta \theta_{2}$ intervals;

- $M_{\{\psi\}}$ for the grouping of azimuth coefficients within $\Delta \phi_{2}$ intervals.

For a given $\Delta \theta_{2}$, a different $M_{\{\theta\}}$ matrix is associated to every discrete $\theta_{2}$ value between 0 and $\theta_{2_{\max }}$ (according to a $\Delta \theta_{2}$ step), leading to $\frac{\theta_{2_{\max }}}{\Delta \theta_{2}}+1$ binary altitude matrices in total; the " +1 " includes the screen apex sector (corresponding to $\theta_{2}=0^{\circ}$ ).

These matrices are calculated from $M_{\theta_{2}}$ and thus depend on $e_{s}$; their coefficients are equal to 1 when related to coefficients in $M_{\theta_{2}}$ comprised within a same $\Delta \theta_{2}$ interval, otherwise equal to 0 . For each discrete $\theta_{2}$ value, $M_{\{\theta\}}\left(\theta_{2}\right)$ thus presents non-zero coefficients only at coordinates corresponding to $M_{\theta_{2}}$ coefficients within the $\theta_{2} \pm \frac{1}{2} \Delta \theta_{2}$ interval.

Binary matrices $M_{\{\psi\}}$ also represent groups of azimuth values within a same $\Delta \phi_{2}$ interval, but need to account for the minimal azimuth shift parameter $S_{\psi}$ too, equal to $\phi_{1} \bmod \Delta \phi_{2}$.

For each shift $S_{\psi}$, a different $M_{\psi_{2}^{\prime}}$ matrix is calculated by adding $S_{\psi}$ to $M_{\psi_{2}}$. The resulting $\psi_{2}^{\prime}$ coefficients can then be grouped for each $M_{\psi_{2}^{\prime}}$ matrix according to $\Delta \phi_{2}$ intervals:

- as the number and configuration of $\psi_{2}^{\prime}$ intervals on the screen depend on 
both $\Delta \phi_{2}$ and $S_{\psi}$, a function of these parameters is needed to delimit the values of the discrete steps $0, \pm \Delta \phi_{2}, \pm 2 \cdot \Delta \phi_{2} \ldots$ around which $\psi_{2}^{\prime}$ values are gathered; this function is named $L_{\psi_{2 \pm}^{\prime}}$ (index "-" for the lower limit on the screen, "+" for the upper limit);

- for a given $\Delta \phi_{2}$ and for each shift $S_{\psi}$, a different $M_{\{\psi\}}$ matrix is associated to every discrete $\psi_{2}^{\prime}$ value between $L_{\psi_{2-}^{\prime}}$ and $L_{\psi_{2}^{+}}$; this leads to $N_{G} \cdot\left(\frac{L_{\psi_{2}^{\prime}}-L_{\psi_{2}^{\prime}}}{\Delta \phi_{2}}+1\right)$ binary azimuth matrices in total, $N_{G}$ being the number of new averaging grids to be constructed for the current BT(R)DF characterization; the "+1" term takes into account that a single sector overlapping two screen positions has to be counted twice;

- these matrices present coefficients equal to 1 when related to coefficients in $M_{\psi_{2}^{\prime}}$ comprised within a same $\Delta \phi_{2}$ interval, otherwise equal to 0. For each discrete $\psi_{2}^{\prime}$ value and each $S_{\psi}, M_{\{\psi\}}\left(\psi_{2}^{\prime}, S_{\psi}\right)$ thus presents non-zero coefficients only at coordinates corresponding to $M_{\psi_{2}^{\prime}}$ coefficients within the $\psi_{2}^{\prime} \pm \frac{1}{2} \Delta \phi_{2}$ interval.

Once all the matrices $M_{\{\theta\}}\left(\theta_{2}\right)$ and $M_{\{\psi\}}\left(\psi_{2}^{\prime}, S_{\psi}\right)$ are available, their elementper-element multiplications according to pairs of values $\theta_{2}$ and $\psi_{2}^{\prime}$ are used to determine the different averaging sectors $\left(\Delta \theta_{2}, \Delta \phi_{2}\right)$ around directions $\left(\theta_{2}, \psi_{2}^{\prime}\right)$ for each shift configuration $S_{\psi}$.

\subsection{Calculation of BT(R)DFs}

To apply Equation (1) at a pixel-level spatial accuracy, i.e. converting the $\frac{L_{\text {screen }}}{E_{1}}$ ratios associated to each pixel after image calibration into their corresponding $B T(R) D F$ data, a correction matrix $M_{C_{\mathrm{BT}(\mathrm{R}) \mathrm{DF}}}$ is constructed, based on Equation (1) and given by Equation (6), from which a floating-point cor- 
rection figure can be created.

$$
M_{\mathrm{C} \text { BT(R)DF }}=\frac{\pi}{\rho_{\text {screen }}} \cdot \frac{M_{d}^{2}}{A \cdot \cos M_{\alpha} \cdot \cos M_{\theta_{2}}}
$$

All operations in Equation (6) are applied on each coefficient individually, matrix $M_{\alpha}$ being calculated from Equation (2) replacing $\theta_{2}$ and $\psi_{2}$ by $M_{\theta_{2}}$ and $M_{\psi_{2}}$ respectively. A different $M_{\mathrm{C}_{\mathrm{BT}(\mathrm{R}) \mathrm{DF}}}$ figure is actually needed for every new combination of parameters $e_{s}$ and $A$, as $e_{s}$ determines the $M_{d}, M_{\theta_{2}}$ and $M_{\alpha}$ coefficients.

An approximate digital representation of matrix $M_{C_{\mathrm{BT}(\mathrm{R}) \mathrm{DF}}}$ is given on Figure 9 , the correction factors range being far too important to be adequately represented by 256 grey levels (they vary from about 37,000 to $1.4 \cdot 10^{21}[$ [] for an arbitrary area $A=1 \mathrm{~cm}^{2}$ ).

Fig. 9. Digital representation of the correction matrix $M_{\mathrm{C}_{\mathrm{BT}(\mathrm{R}) \mathrm{DF}}}$, compensating distance and light tilting effects to convert $\frac{L_{\mathrm{screen}}}{E_{1}}$ ratios into $B T(R) D F$ values with a pixel-level spatial accuracy.

Once the image pixels are fully calibrated into BT(R)DFs, the image is imported as a matrix into Matlab ${ }^{\circledR}$ and the coefficients averaged according to intervals $\left(\Delta \theta_{2}, \Delta \phi_{2}\right)$ resulting from the multiplication of matrices $M_{\{\theta\}}\left(\theta_{2}\right)$ and $M_{\{\psi\}}\left(\psi_{2}^{\prime}, S_{\psi}\right)$ for every combination of $\theta_{2}$ and $\psi_{2}^{\prime}$ and the current shift configuration $S_{\psi}$.

This leads at each run to a matrix composed of BT(R)DF values only for the elements concerned by the current angular sector (and of zeros elsewhere), from which a mean BT(R)DF can be deduced. 
When an angular sector overlaps adjacent screen positions (generally two, six for the $\left(\theta_{2}, \phi_{2}\right)=\left(0^{\circ}, 0^{\circ}\right)$ sector $)$, a special treatment is required as the latter will correspond to the same angular couple $\left(\theta_{2}, \phi_{2}\right)$ but to a different screen position and $\psi_{2}$ value. For example, on Figure ??, the sector $\left(75^{\circ}, 45^{\circ}\right)$ appears on both screen positions 1 and 2 , where it respectively corresponds to $\psi_{2}$ values $30^{\circ}$ and $-30^{\circ}$. Besides, a weighing is then necessary to deduce the mean value of the full sector: although the image pixels represent unequal portions of the full screen area because of perspective and image distortion effects, it was checked that no perceptible modification in $\mathrm{BT}(\mathrm{R}) \mathrm{DF}$ data was observed when associating graded weights to each of them; thus, a (sub)sector's area is simply given by the number of comprised pixels.

\subsection{Procedure validation and optimization}

As Image-Pro Plus ${ }^{\circledR}$ proposes a very efficient method for analyzing an image within defined groups of pixels, this method was adapted to take advantage of it, adding several operations on the $M_{\{\theta\}}\left(\theta_{2}\right)$ and $M_{\{\psi\}}\left(\psi_{2}^{\prime}, S_{\psi}\right)$ matrices to create greyscale level patterns out of them. The latter are used by ImagePro Plus ${ }^{\circledR}$ as supports for outlining the pixels groups properly. Examples are shown on Figures 2(a) and 2(b); their construction is explained below.

The image and data processing procedure presented in this section has been tested successfully, proving reliability and unrestricted possibilities of adaptation to the sample properties and averaging grid intervals.

It has though been observed that such a program increases the time needed to perform a measurement: whereas a few minutes are sufficient to achieve a 
$\mathrm{BT}(\mathrm{R}) \mathrm{DF}$ characterization for one incident direction with a fixed geometric calibration, this particular data processing adds about 15 to 20 seconds at each screen position, inducing a doubling of the required processing time (these values actually strongly depend on the computer properties as well as on the chosen grid, reduced with large angular intervals).

Such amounts of time certainly remain appreciably inferior to the ones required for conventional, step-by-step goniophotometric measurements, which are of several hours, but nevertheless present a non-negligible increase when considered in a relative way. Therefore, an optimization of the described procedure has been sought, calling on a combination of two softwares: MATLAB ${ }^{\circledR}$ for the matrix calculations necessary to create the different discretization grids, and the image acquisition software IMAGE-PRO PLUS ${ }^{\circledR}$, also allowing image processing and operations, for a considerable acceleration of the $\mathrm{BT}(\mathrm{R}) \mathrm{DF}$ extraction according to a specific grid.

The optimized image and data processing (15) has been experimented in usual and critical configurations and has proven to be very reliable and stable, providing efficient and consistent results in the discretization sectors determination and $\mathrm{BT}(\mathrm{R}) \mathrm{DF}$ assessment, and inducing no lengthening in the measurement process when compared to a fixed geometry.

\section{Conclusions}

In this paper, a new method for adapting geometric calibration for digital image-based goniophotometric measurements with variable referential is described. This method relies on the use of matrix calculations that provide an 
appreciable flexibility in the output resolution and a precise adjustment of the coordinate system according to the reference frame parameters: such reciprocal transformations from images to matrices, considered as ( $\mathrm{n} \times \mathrm{m})$ arrays of data, allow accurate geometric adaptations and an appropriate and flexible grouping of pixel-level BT(R)DFs into averaging sectors.

The procedure was developed within the framework of BT(R)DF measurements performed with a bidirectional goniophotometer based on digital imaging techniques. It proved to be very efficient, providing reliable results without requiring any significant additional time amount for a BT(R)DF assessment, that remains at about a minute per incident direction thanks to a programming combination of MATLAB ${ }^{\circledR}$ and IMAGE-PRO PLUS ${ }^{\circledR}$. It was moreover successfully tested even for critical configurations, like extreme output resolutions or important referential transformations.

Having a full control at the pixel level allows an extended flexibility in imagebased photometric measurements, which can become particularly interesting for spatial investigations that have to be adapted to specific needs (polar or solid angles, grid of variable meshes), for output resolution changes, and, in the case of bidirectional goniophotometric measurements, for handling blind sectors caused by the incident beam region when dealing with reflection distributions (15).

\section{Acknowledgements}

This work was supported by the Swiss Federal Institute of Technology (EPFL) and the Commission for Technology and Innovation (CTI). The author wishes 
to thank Dr. Laurent Michel for his helpful suggestions about the use of matrices to describe an image-based geometric system.

\section{References}

[1] Andersen M, Michel L, Roecker C, Scartezzini J-L. Experimental assessment of bi-directional transmission distribution functions using digital imaging techniques. Energy and Buildings 2001;33(5):417-431.

[2] Ashdown I. Near-Field Photometry: A new approach. Journal of the Illuminating Engineering Society 1993;22(1):163-180.

[3] Berrutto V, Fontoynont M. Applications of CCD cameras to lighting research: Review and extension to the measurement of glare indices. Proceedings CIE 119, 23rd Session, New Delhi 1995:192-195.

[4] Berrutto V. Métrologie de la qualité visuelle des ambiances lumineuses: Application à l'éclairage des bureaux. PhD thesis, Université de Savoie, Chambéry 1996.

[5] Karner KF, Mayer H, Gervautz M. An Image based Measurement System for Anisotropic Reflection. Computer Graphics Forum 1996;15(3):119128.

[6] Lewin I, O'Farrell J. Luminaire Photometry Using Video Camera. Journal of the Illuminating Engineering Society 1999;28(1):57-63.

[7] Marschner SR, Westin SH, Lafortune EPF, Torrance KE. Image-based bidirectional reflectance distribution function measurement. Applied Optics 2000;39(16):2592-2600.

[8] Michel L. Méthode expérimentale d'évaluation des performances lumineuses de bâtiments. PhD thesis, EPFL, Lausanne 1999.

[9] Song KD, Degelman LO, Boyer LL. Determining Daylighting Parame- 
ters by a Luminance Mapping System and Scale Models. Journal of the Illuminating Engineering Society 1994;23(1):65-75.

[10] Tansley BW, Houser KW, Pasini IC. The IQCam Digital Image Photometer System: Principles of Operation and Comparative Performance. Journal of the Illuminating Engineering Society 1999;28(1):182-200.

[11] Ward GJ. Measuring and Modeling Anisotropic Reflection. Computer Graphics 1992;26(2):265-272.

[12] Whitehead LA, Kan P, Lui K, Jacob S. Near Field Photometry of Prism Light Guide Luminaires Using a CCD Camera. Journal of the Illuminating Engineering Society 1999;28(2):3-9.

[13] Cardenas-Garcia JF, Yao HG, Zheng S. 3D Reconstruction of Objects Using Stereo Imaging. Optics and Lasers in Engineering 1995;22(3):193213.

[14] Tao CV. Semi-automated object measurement using multiple-image matching from mobile mapping image sequences. Photogrammetric Engineering and Remote Sensing 2000;66(12):1477-1485.

[15] Andersen M. Innovative Bidirectional Video-Goniophotometer for Advanced Fenestration Systems. PhD thesis, EPFL, Lausanne 2004.

[16] Andersen M, Rubin M, Scartezzini J-L. Comparison between ray-tracing simulations and bi-directional transmission measurements on prismatic glazing. Solar Energy 2003;74(2):157-173. 


\section{List of Figures}

1 Assessment method and polar coordinates of the Bidirectional Transmission Distribution Function (defined likewise in reflection).

2 Division of the screen measurement area into discretisation sectors, differentiated by grey level gaps.

3 Relationship between polar co-ordinates $\left(\theta_{2}, \psi_{2}\right)$ and reference coordinates $\left(\theta_{2_{\text {Ref }}}, \psi_{2}\right)$, combined to the associated cartesian screen co-ordinates $(x, y)$.

4 Digital representation of reference matrices (for a quantitative analysis, they can be compared against the contour plots of Figures 2(a) and 2(b).

5 Orthogonal projection view of the sample orientation superimposed on the six screen positions.

6 Superimposed averaging grids with $\left(\Delta \theta_{2}, \Delta \phi_{2}\right)=\left(10^{\circ}, 15^{\circ}\right)$ for $e_{s}=7.5 \mathrm{~cm}$ (sectors borders) and $e_{s}=0$ (greyscale level pattern).

$7 \quad$ Schematised global averaging grid for $\left(\Delta \theta_{2}, \Delta \phi_{2}\right)=\left(15^{\circ}, 15^{\circ}\right)$ and sectors' polar coordinates.

8 Superimposed averaging grids with $\left(\Delta \theta_{2}, \Delta \phi_{2}\right)=\left(10^{\circ}, 15^{\circ}\right)$ for $\phi_{1}=50^{\circ}$ (sectors borders) and $\phi_{1}=0^{\circ}$ (greyscale level pattern), displaying a $5^{\circ}$ shift in azimuth.

9 Digital representation of the correction matrix $M_{\mathrm{C}_{\mathrm{BT}(\mathrm{R}) \mathrm{DF}}}$, compensating distance and light tilting effects to convert $\frac{L_{\text {screen }}}{E_{1}}$ ratios into $B T(R) D F$ values with a pixel-level spatial accuracy. 


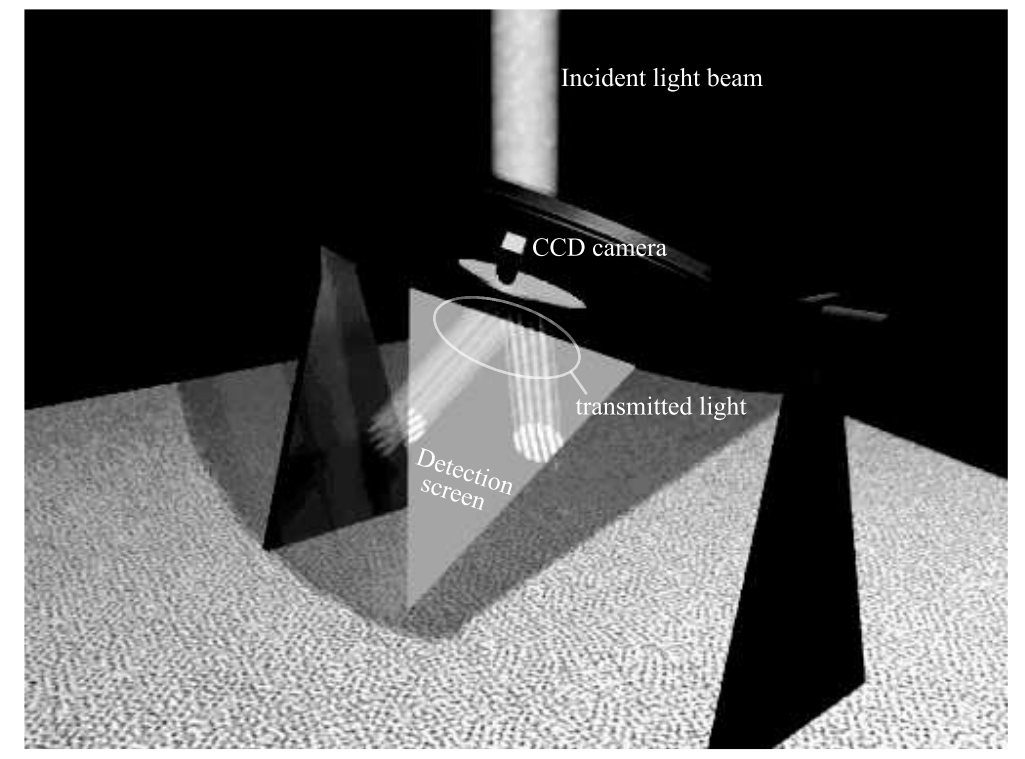

(a) Detection of the transmitted light flux with CCD camera

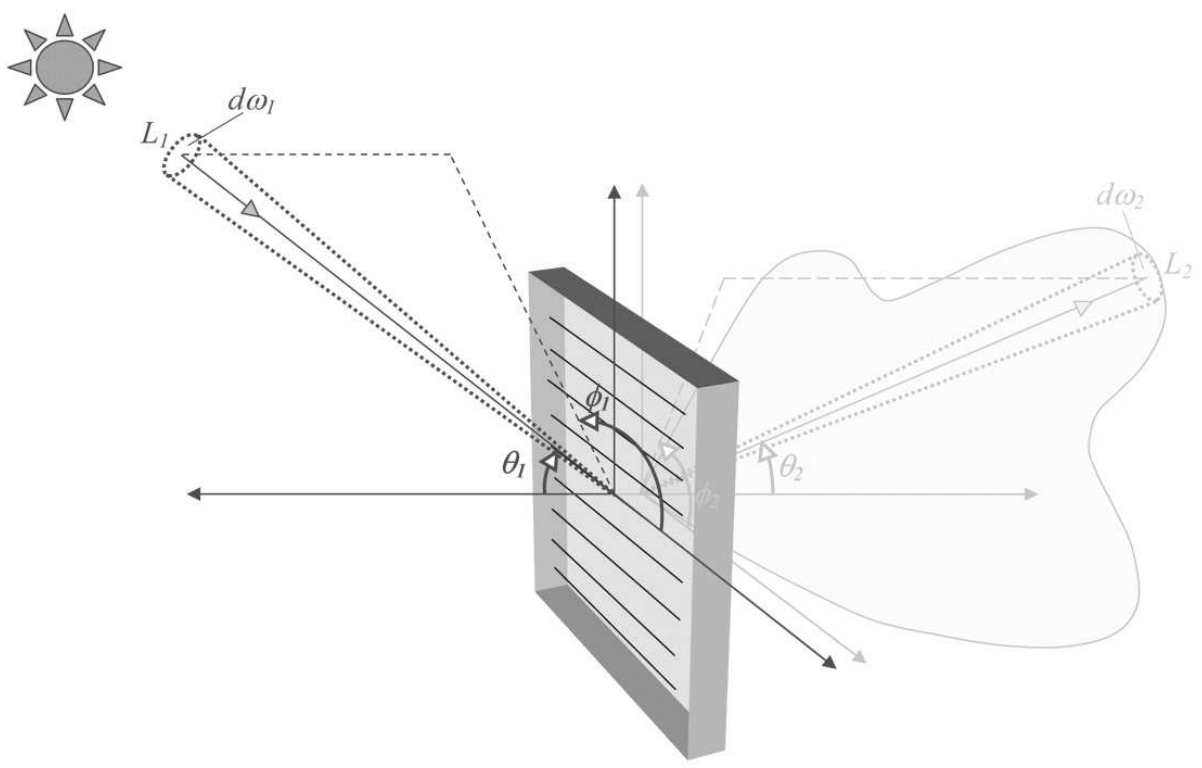

(b) BTDF referential definition

Fig. 1. Assessment method and polar coordinates of the Bidirectional Transmission Distribution Function (defined likewise in reflection). 


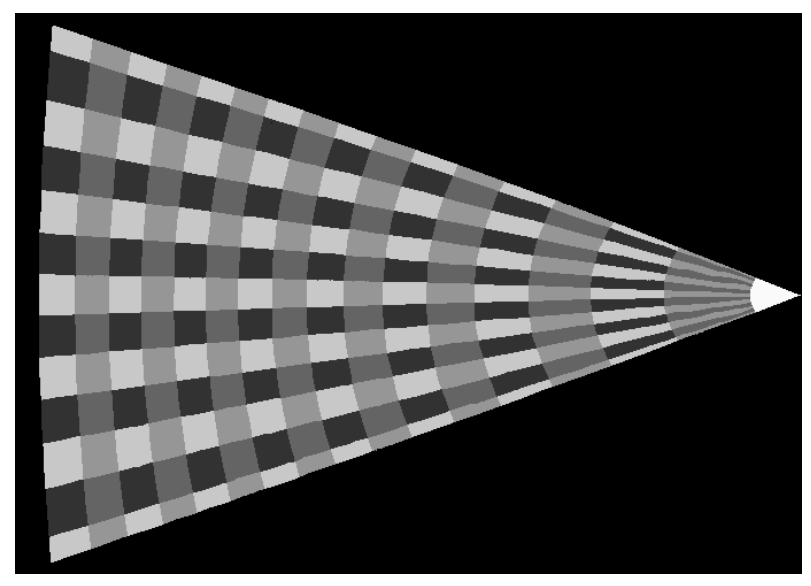

(a) Averaging intervals $\left(\Delta \theta_{2}, \Delta \phi_{2}\right)=\left(5^{\circ}, 5^{\circ}\right)$

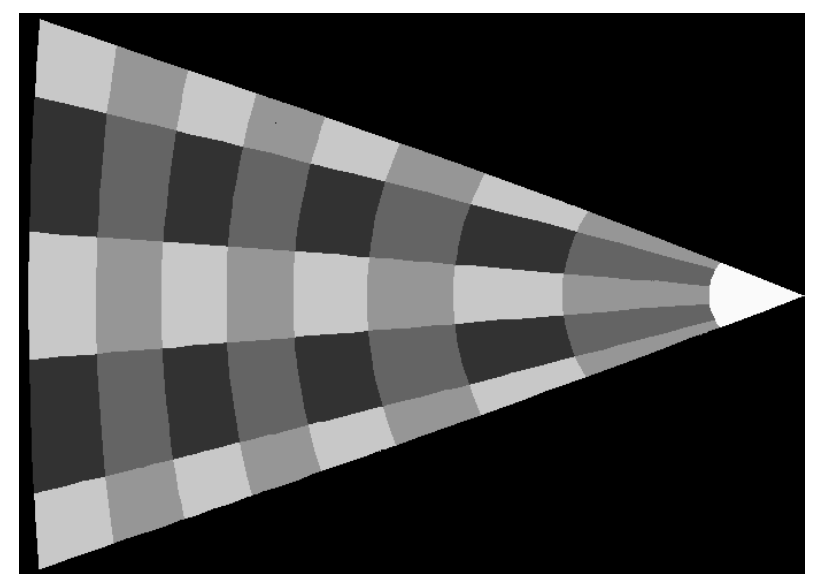

(b) Averaging intervals $\left(\Delta \theta_{2}, \Delta \phi_{2}\right)=$ $\left(10^{\circ}, 15^{\circ}\right)$

Fig. 2. Division of the screen measurement area into discretisation sectors, differentiated by grey level gaps. 


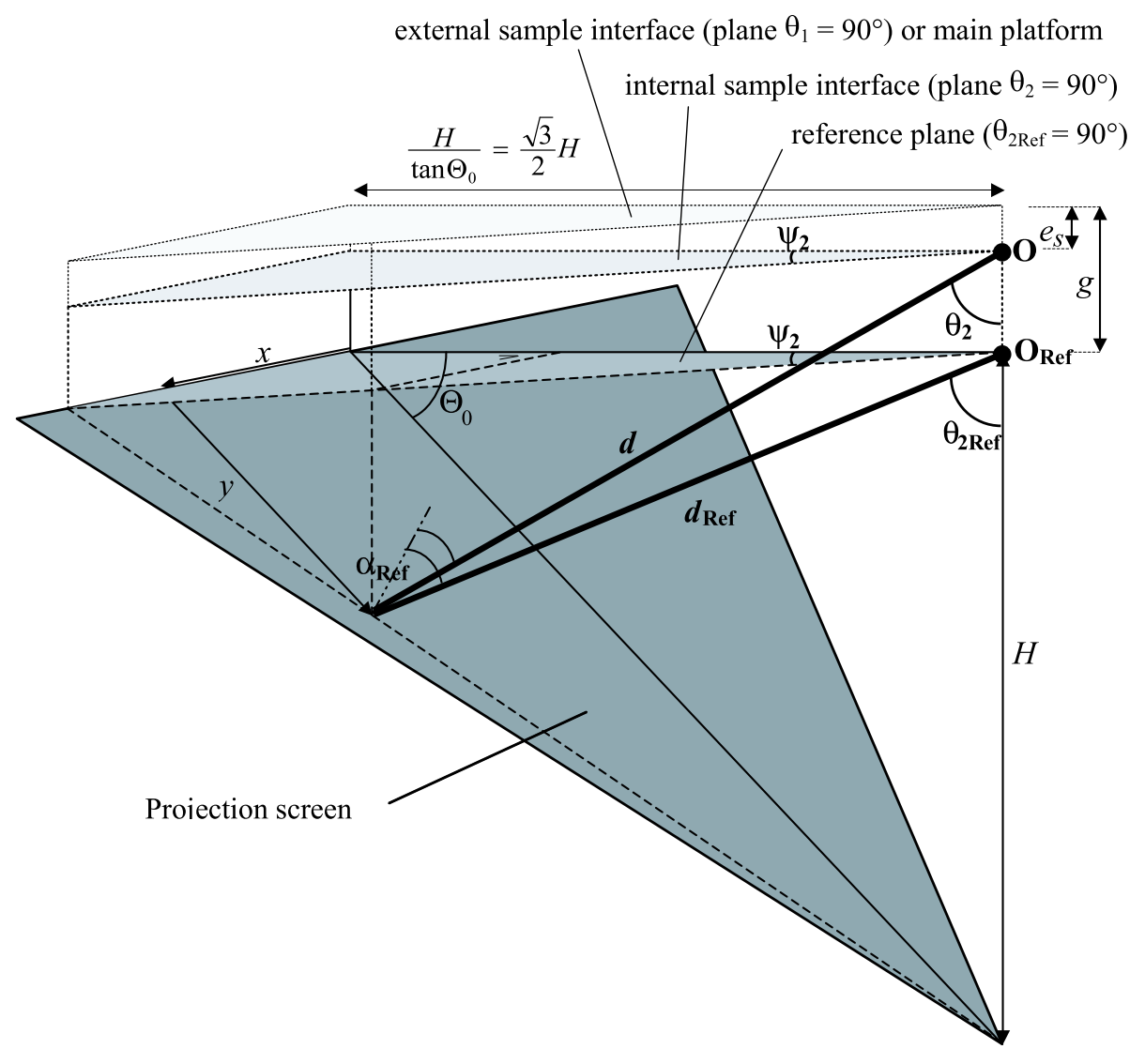

Fig. 3. Relationship between polar co-ordinates $\left(\theta_{2}, \psi_{2}\right)$ and reference coordinates $\left(\theta_{2_{\text {Ref }}}, \psi_{2}\right)$, combined to the associated cartesian screen co-ordinates $(x, y)$. 


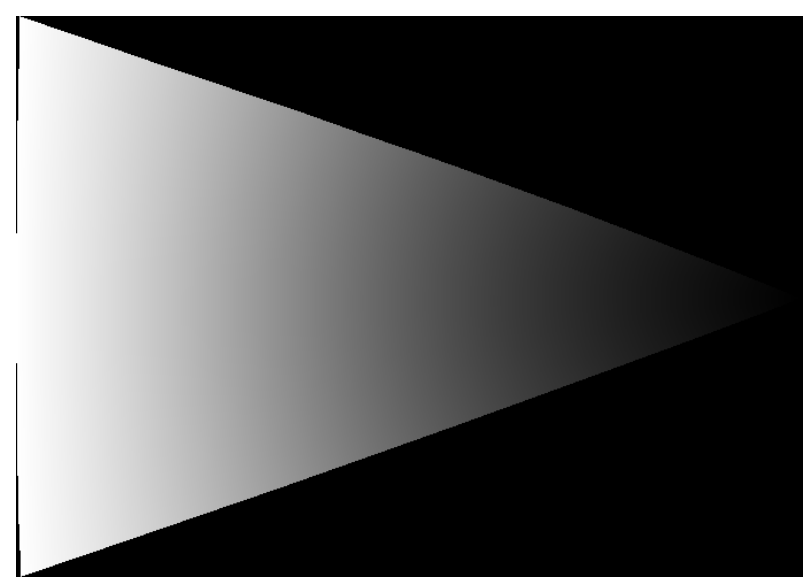

(a) Matrix $M_{\theta_{2} \text { Ref }}$

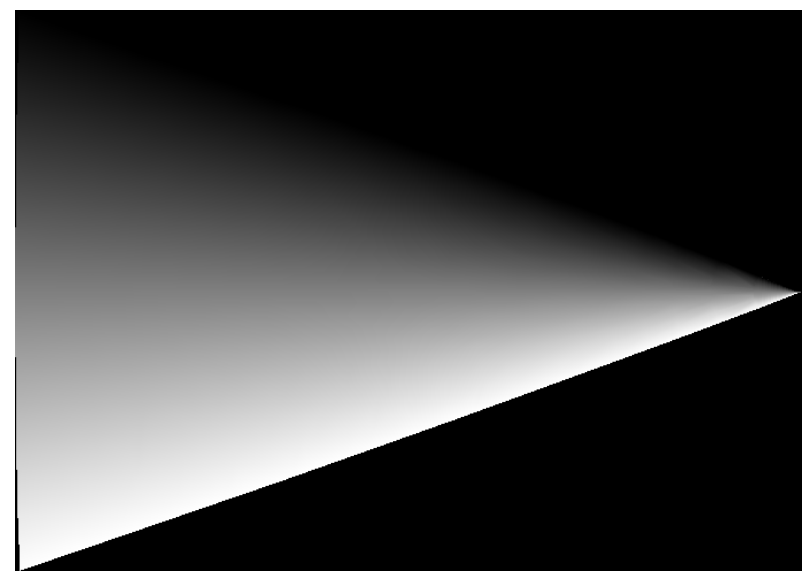

(b) Matrix $M_{\psi_{2}}$

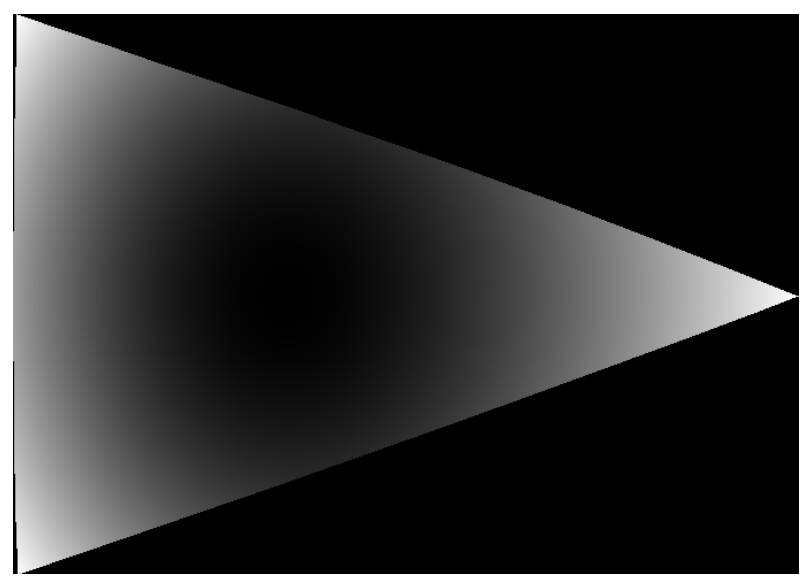

(c) Matrix $M_{d_{\mathrm{Ref}}}$

Fig. 4. Digital representation of reference matrices (for a quantitative analysis, they can be compared against the contour plots of Figures 2(a) and 2(b). 


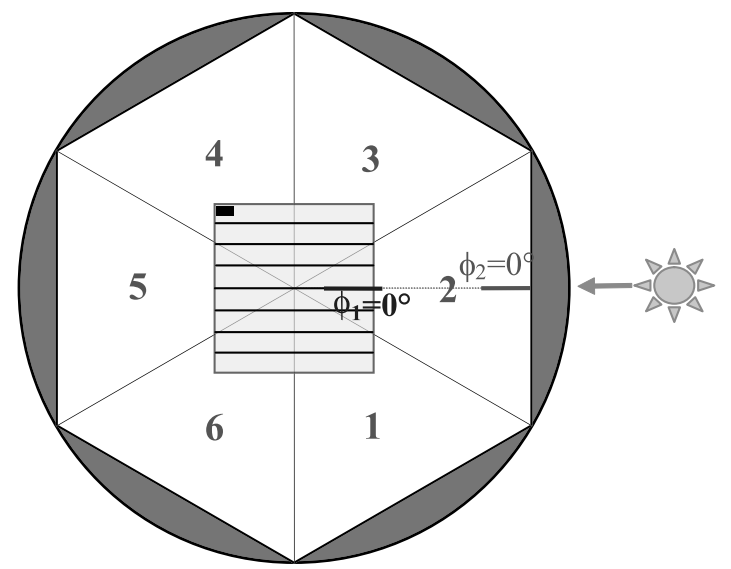

(a) $\phi_{1}=0^{\circ}$ i.e. default sample orientation

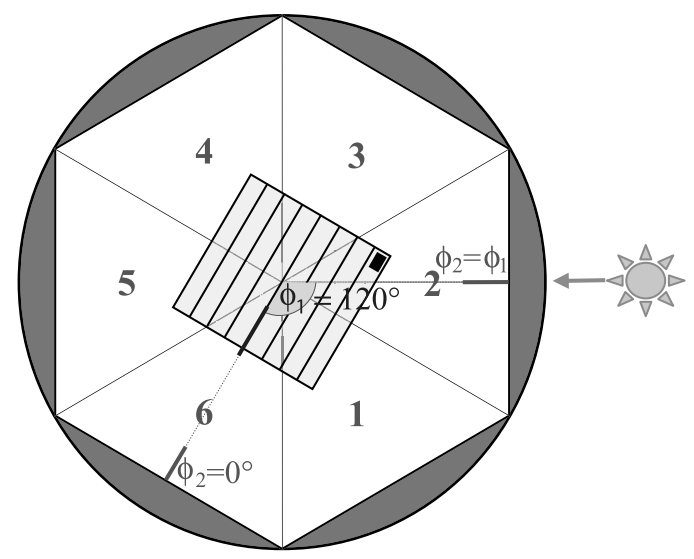

(b) $\phi_{1}=120^{\circ}$, sample and azimuth origin axis have been rotated

Fig. 5. Orthogonal projection view of the sample orientation superimposed on the six screen positions. 


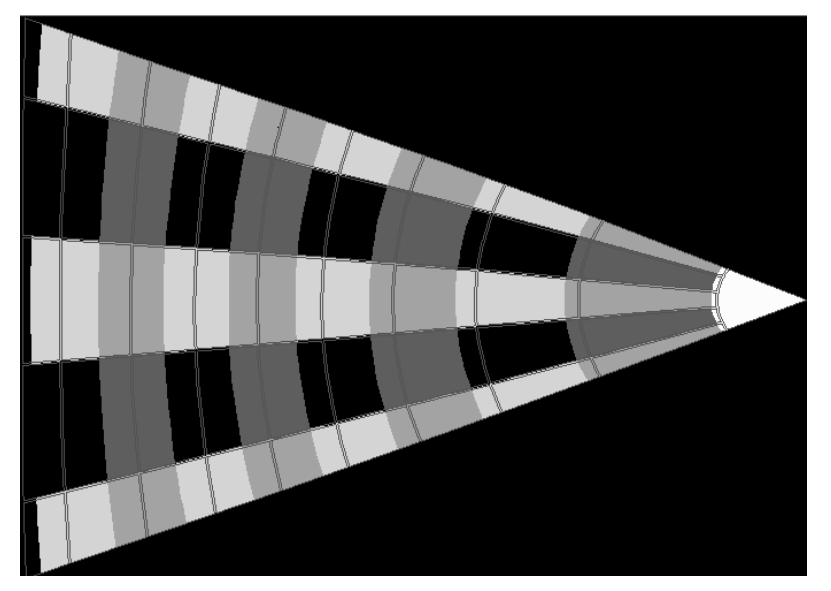

Fig. 6. Superimposed averaging grids with $\left(\Delta \theta_{2}, \Delta \phi_{2}\right)=\left(10^{\circ}, 15^{\circ}\right)$ for $e_{s}=7.5 \mathrm{~cm}$ (sectors borders) and $e_{s}=0$ (greyscale level pattern). 


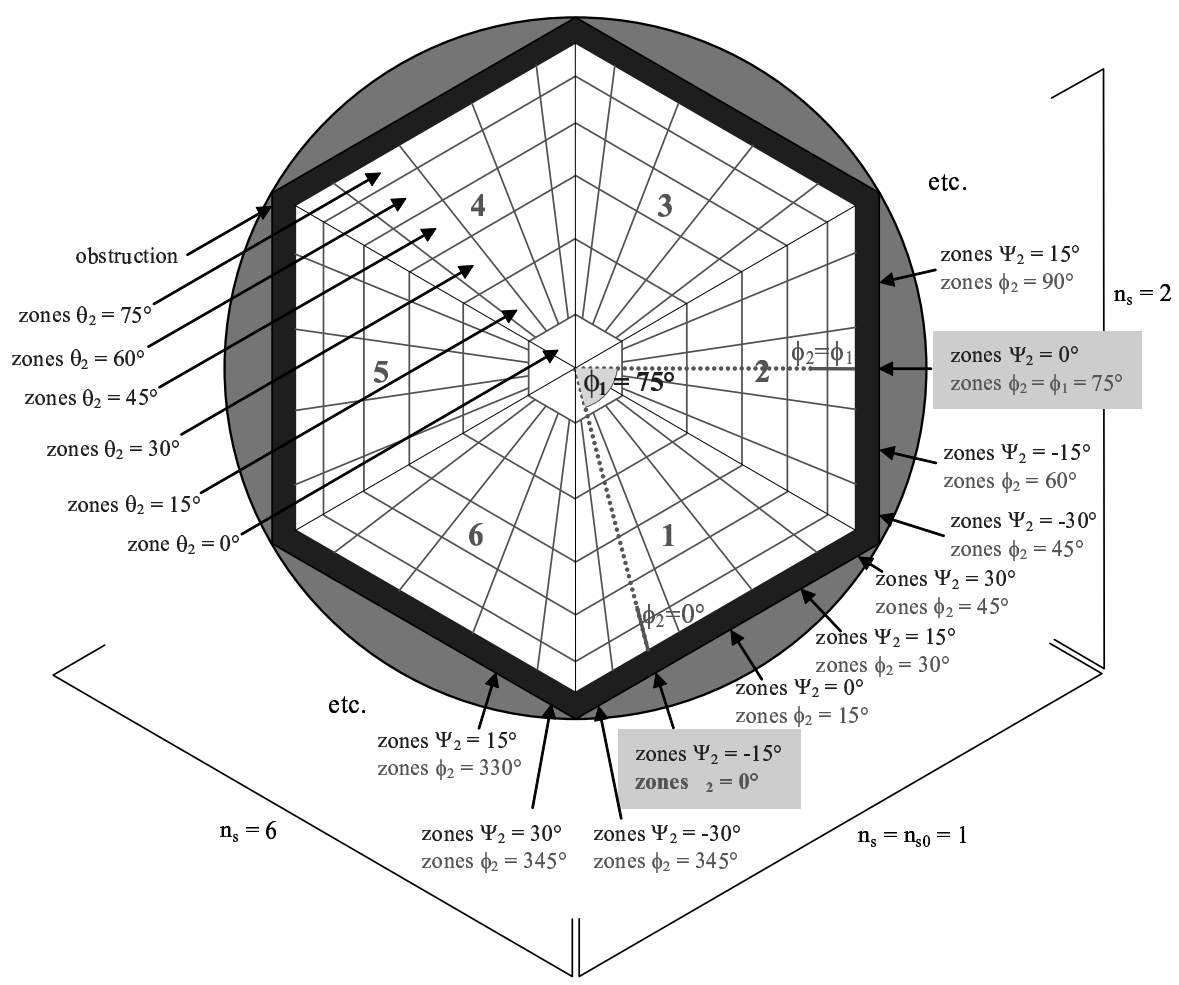

(a) $\phi_{1}=75^{\circ}=N \cdot \Delta \phi_{2}$ with $N=5$

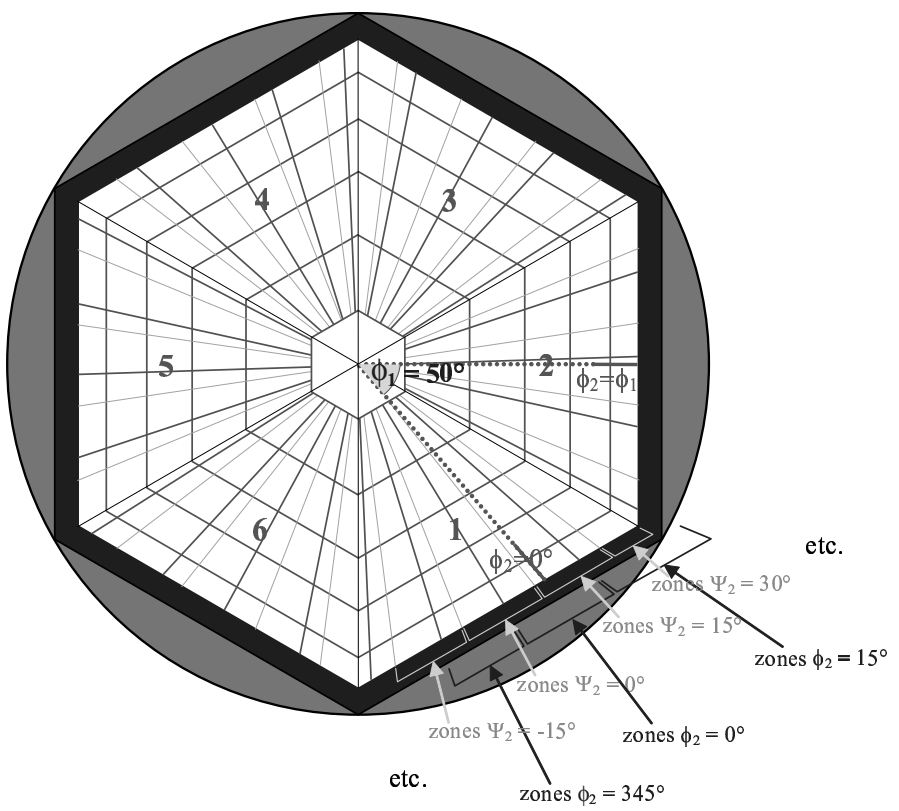

(b) $\phi_{1}=50^{\circ}$ (grid superimposed on the $\phi_{1}=N \cdot \Delta \phi_{2}$ default grid)

Fig. 7. Schematised global averaging grid for $\left(\Delta \theta_{2}, \Delta \phi_{2}\right)=\left(15^{\circ}, 15^{\circ}\right)$ and sectors' polar coordinates. 


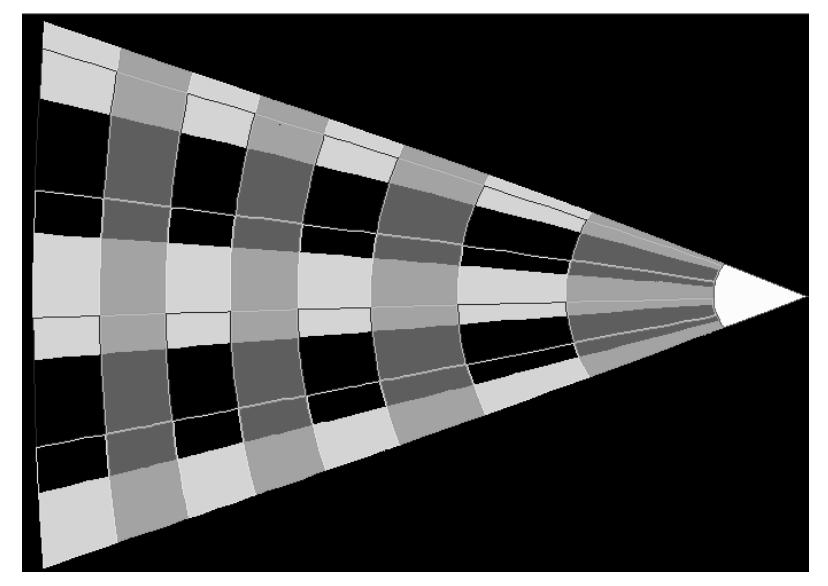

Fig. 8. Superimposed averaging grids with $\left(\Delta \theta_{2}, \Delta \phi_{2}\right)=\left(10^{\circ}, 15^{\circ}\right)$ for $\phi_{1}=50^{\circ}$ (sectors borders) and $\phi_{1}=0^{\circ}$ (greyscale level pattern), displaying a $5^{\circ}$ shift in azimuth. 


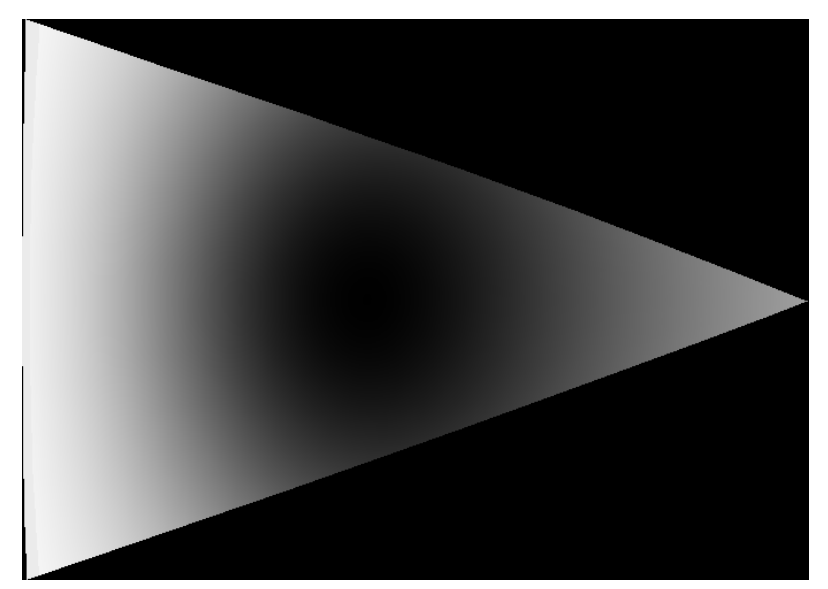

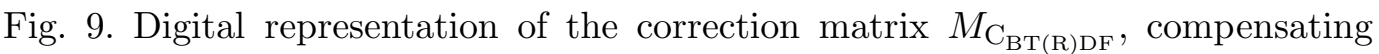
distance and light tilting effects to convert $\frac{L_{\text {screen }}}{E_{1}}$ ratios into $B T(R) D F$ values with a pixel-level spatial accuracy. 